\title{
Qualidade da informação disponível na internet sobre depressão e antidepressivos
}

\author{
Quality of information available on the internet about depression and antidepressants \\ Calidad de la información disponible en Internet sobre depresión y antidepresivos
}

\section{Resumo}

A depressão é um transtorno mental caracterizado por tristeza persistente e pela perda de interesse em atividades que normalmente são prazerosas, acompanhadas da incapacidade de realizar atividades diárias, durante pelo menos duas semanas. A qualidade das informações oferecidas na internet tem sido objeto de avaliação em diversas áreas e especialmente, na de saúde. E, neste cenário, torna-se cada vez mais importante investigar a qualidade da informação a que a população tem acesso sobre sua saúde e sobre medicamentos. O objetivo deste trabalho foi avaliar a qualidade das informações prestadas por sites de saúde na língua portuguesa referentes à depressão e ao uso de medicamentos para seu tratamento. Trata-se de um estudo transversal, dividido em duas etapas: avaliação da qualidade das informações globais em sites para a saúde relacionados à depressão e avaliação da qualidade da informação sobre utilização de antidepressivos. Foram analisados 50 sites. Na avaliação da qualidade das informações globais, nenhum dos 50 sites analisados foi considerado com qualidade alta, $32 \%$ foram classificados com qualidade moderada, $64 \%$ com qualidade ruim e $4 \%$ com qualidade muito ruim. Na avaliação da qualidade da informação sobre depressão, $46 \%$ foram classificados com qualidade muito ruim, $40 \%$ com qualidade ruim, $6 \%$ com qualidade moderada e $8 \%$ com qualidade alta. Os resultados mostram uma baixa qualidade da informação disponibilizada na internet sobre antidepressivos e depressão, sugerindo a necessidade de disponibilizar e oferecer aos usuários informações de maior qualidade na internet. Palavras-chave: Qualidade; Informação; Antidepressivos; Depressão.

\begin{abstract}
Depression is a mental disorder characterized by persistent sadness and loss of interest in activities that are normally pleasurable, accompanied by an inability to perform daily activities for at least two weeks. The quality of information offered on the internet has been the object of evaluation in several areas, especially in health. And, in this scenario, it becomes increasingly important to investigate the quality of information that the population has access to about their health and medicines. The objective of this study was to evaluate the quality of information provided by health websites in Portuguese regarding depression and the use of medication for its treatment. This is a cross-sectional study, divided into two stages: assessment of the quality of global information on depression-related health websites and assessment of the quality of information on the use of antidepressants. 50 sites were analyzed. In assessing the quality of global information, none of the 50 sites analyzed was considered to be of high quality, $32 \%$ were classified as moderate quality, $64 \%$ as poor quality and $4 \%$ as very poor quality. In assessing the quality of information about depression, $46 \%$ were classified as very poor quality, $40 \%$ as poor quality, $6 \%$ as moderate quality and $8 \%$ as high quality. The results show a low quality of information available on the internet about antidepressants and depression, suggesting the need to make available and offer users higher quality information on the internet.
\end{abstract}

Keywords: Quality; Information; Antidepressants; Depression. 


\begin{abstract}
Resumen
La depresión es un trastorno mental que se caracteriza por una tristeza persistente y una pérdida de interés en actividades que normalmente son placenteras, acompañada de una incapacidad para realizar las actividades diarias durante al menos dos semanas. La calidad de la información ofrecida en Internet ha sido objeto de evaluación en varios ámbitos, especialmente en salud. Y, en este escenario, se vuelve cada vez más importante investigar la calidad de la información a la que tiene acceso la población sobre su salud y medicamentos. El objetivo de este estudio fue evaluar la calidad de la información proporcionada por los sitios web de salud en portugués sobre la depresión y el uso de medicamentos para su tratamiento. Se trata de un estudio transversal, dividido en dos etapas: evaluación de la calidad de la información global en sitios web de salud relacionados con la depresión y evaluación de la calidad de la información sobre el uso de antidepresivos. Se analizaron 50 sitios. Al evaluar la calidad de la información global, ninguno de los 50 sitios analizados se consideró de alta calidad, el $32 \%$ se clasificó como de calidad moderada, el $64 \%$ como de mala calidad y el $4 \%$ como de muy mala calidad. Al evaluar la calidad de la información sobre la depresión, el 46\% se clasificó como de muy mala calidad, el $40 \%$ como de mala calidad, el $6 \%$ como de calidad moderada y el $8 \%$ como de alta calidad. Los resultados muestran una baja calidad de la información disponible en Internet sobre antidepresivos y depresión, lo que sugiere la necesidad de poner a disposición y ofrecer a los usuarios información de mayor calidad en Internet.
\end{abstract}

Palabras clave: Calidad; Información; Antidepresivos; Depresión.

\title{
1.Introdução
}

A Internet é a maior ferramenta de busca de informação na atualidade, sendo o principal local para busca de informação tanto de leigos como de profissionais da área de saúde. A partir de sua popularização, a pesquisa bibliográfica sofreu mudanças, antes restritas aos livros, enciclopédias e revistas, ocorrendo também um rompimento de barreiras geográficas, culturais e sociais (Hirata et al., 2010).

Além disso, a internet permitiu aos usuários acesso à informação sobre saúde de forma fácil e rápida. Estima-se que 70 \% dos americanos, por exemplo, buscam informação sobre saúde na internet (Varady et al., 2018).

A informação sobre saúde e doença está acessível na internet, mas muitas vezes ela é incompleta, contraditória, incorreta ou até fraudulenta. Por esta razão, o cidadão comum muitas vezes tem dificuldade de distinguir, por exemplo, o certo do enganoso ou o inédito do tradicional (Garbin et al., 2008; Ribeiro et al., 2021). Com relativa facilidade, qualquer pessoa pode postar conteúdo, em todo o mundo, na forma de livros, conselhos, testemunho de curas milagrosas ou serviços clínicos (Lissman et al., 2001). Muitas vezes os pacientes leigos têm dificuldade com a linguagem médica e com a incerteza advinda da multiplicidade de informações. Somam-se a isso os problemas decorrentes do estímulo à automedicação (Garbin et al., 2008).

Um requisito mínimo para um site de informações de saúde ter alta qualidade deve ser disponibilizar conteúdo baseado em evidências científicas, ou seja, que seu conteúdo e recomendações sejam consistentes com as evidências de uma revisão sistemática da literatura médica disponível (Griffiths et al., 2005).

A Fundação Health On the Net $(H O N)$, uma organização não governamental sediada na Suíça, é uma das mais antigas e conhecidas iniciativas de avaliação da qualidade da informação sobre saúde na internet. A HON atribui um selo de qualidade para os sites de saúde que estejam em conformidade com os princípios do HONCode. No Brasil, o Conselho Regional de Medicina de São Paulo (Cremesp), elaborou o Guia de ética para sites de medicina e saúde na internet, em que define princípios éticos e estabelece padrões mínimos de qualidade, segurança e confiabilidade para sites de medicina e saúde, e semelhantes em alguns pontos à proposta da HON Foundation ${ }^{6}$.

Tendo em vista a grande dificuldade de acesso aos serviços de saúde, contrapondo-se às facilidades no mundo virtual e o hábito da automedicação pela população brasileira, o acesso à internet costuma preceder a consulta médica. Assim, existe preocupação crescente entre os profissionais da saúde em relação à qualidade e à confiabilidade das informações veiculadas (Hirata et al., 2010).

A depressão é a alteração afetiva mais estudada e falada na atualidade, levando em consideração a pandemia causada pela COVID-19. As medidas de controle, de distanciamento e isolamento social afetam a população de forma significativa no componente da saúde mental (Griffiths et al., 2005). 
Classificada como um transtorno de humor, ela vem comandar as atitudes dos indivíduos modificando a percepção de si mesmos, e passam a enxergar suas problemáticas como grandes catástrofes. Tratada como a doença da sociedade moderna, a depressão tem características que podem traduzir uma patologia grave ou ser apenas mais um sintoma do sujeito diante de uma situação real da vida (Esteves et al., 2006).

Assim, considerando tanto o papel da depressão no cenário da saúde pública quanto o papel da internet na busca e disseminação de informação em saúde, é importante avaliar a qualidade da informação disponibilizada na internet sobre a depressão em si e sobre os medicamentos utilizados para seu tratamento.

\section{Metodologia}

\subsection{Caracterização do estudo}

Trata-se de um estudo qualitativo e transversal, dividido em duas etapas: avaliação da qualidade global de sites relacionados à depressão e avaliação da qualidade da informação sobre utilização de antidepressivos e depressão.

\subsection{Ferramentas de avaliação}

Trata-se de um estudo quantitativo, descritivo, transversal (Pereira et al., 2018), de avaliação da qualidade das informações globais nos sites realizado a partir de adaptação das ferramentas descritas por Mendonça et al. (2015) que propõem a aplicação de três dimensões para a avaliação da qualidade da informação em sites de saúde no Brasil: conteúdo, técnica e design.

A dimensão conteúdo engloba abrangência, acurácia e inteligibilidade dos dados divulgados; a dimensão técnica tem como objetivo avaliar a credibilidade, segurança e privacidade, representa o nível de transparência da informação e por último a dimensão design que avalia a usabilidade e acessibilidade dos usuários dos sites, representa o nível interatividade do leitor com o site. Para cada uma das dimensões foram descritos grupos de perguntas que permitem avaliá-las. A dimensão conteúdo é constituída por 18 perguntas. A técnica conta com 40 perguntas, duas das quais, relacionadas à privacidade das informações coletadas, não foram utilizadas devido à dificuldade de análise pelos avaliadores. Por fim, a dimensão design é composta por 22 perguntas. Para cada item avaliado foi aplicada a pontuação na escala de zero (0) a dois (2), onde zero é a ausência das informações e 2 é a totalidade das informações (Mendonça et al., 2015).

Num segundo momento, a ferramenta proposta por Griffiths et al. (2005) foi utilizada para avaliar a qualidade do conteúdo da informação propriamente dita, com ênfase em depressão. Esta ferramenta é composta por 20 perguntas sobre o escopo e a correção dos conceitos apresentados e utiliza pontuação própria, onde zero indica a ausência daquele item e um, a presença daquele item (Quadro 2).

\subsection{Critérios de busca}

A seleção dos sites foi feita por meio das ferramentas de busca Google (http:// www.google.com.br) e Yahoo (http:// www.yahoo.com.br). De acordo com Caldeira (2015), a ferramenta de busca Google possui 91,78 \% de participação no mercado global, e ocupa a posição líder entre os buscadores mais utilizados no Brasil com 85,45 \% de participação dos internautas. O Filtro de busca avançada não foi aplicado, para que haja uma aproximação do modo de pesquisa de um usuário.

Foram feitas duas buscas em cada buscador, Google e Yahoo, com a combinação através de operadores booleanos de palavras coloquiais que se aproximassem daquelas usadas por público leigo "antidepressivos" AND (medicamentos OR remédios) e depressão AND tratamento. As buscas foram realizadas considerando apenas a língua portuguesa do Brasil, pois a intenção do trabalho foi analisar sites brasileiros. 
Não foram considerados sites que redirecionam para formatos: pdf, .doc, .docx e vídeos; sites escritos em língua estrangeira; sites que solicitam cadastro, pagamento ou assinatura para leitura do conteúdo; Bulários; sites de drogarias e vendas online; sites não específicos para depressão.

Por meio da busca realizada no Google no dia 20 de abril de 2020, foram encontrados 136 sites. Aplicando os critérios de exclusão, 64 sites foram excluídos, totalizando 72 sites selecionados para avaliação. A mesma busca foi realizada na ferramenta de busca Yahoo, no dia 29 de abril de 2020, sendo encontrados 102 sites. Aplicando os critérios de exclusão, 69 sites foram excluídos, restando 33 sites para avaliação.

Após a retirada dos sites duplicados, foi realizado um cálculo de proporcionalidade para a definição dos 50 sites a serem analisados. Assim foram avaliados os primeiros 34 sites do buscador Google e os primeiros 16 sites do buscador Yahoo.

Para realizar a busca dos sites foi utilizado um computador particular novo, ainda sem dados de navegação no histórico de pesquisa. A utilização deste recurso teve o intuito de reduzir a personalização dos resultados, devido à existência de filtros invisíveis presentes nos sites de busca que poderiam influenciar nos resultados da pesquisa, resultando em um banco de dados com características de pesquisas prévias (Caldeira et al., 2015).

Cada site foi avaliado por dois indivíduos. a nota final foi obtida pela média das pontuações dos dois avaliadores.

\subsection{Análise dos dados}

A análise dos dados do conteúdo presente nos sites foi realizada de acordo com a proposta de Charnock et al. (1999):

-Zero (0) - representa a ausência de determinado item nos sítios analisado;

- Um (1) - representa a presença parcial de determinado item nos sítios analisado

- Dois (2) - representa a presença total do item avaliado nos sítios.

Desta forma o somatório das repostas presentes nos sites permitiu estratificá-los em níveis de qualidade por meio de quartis:

- Qualidade alta - $\geq 75 \%$ - a dimensão analisada (conteúdo, técnico e farmacológico) apresenta classificação (2) para a maioria dos critérios. Indicará que a publicação é de boa qualidade, isto é, uma fonte útil e adequada de informações sobre o tratamento.

- Qualidade moderada: $<75 \geq 50 \%$ - a dimensão analisada (conteúdo, técnico e farmacológico) apresenta classificação (1) para a maioria dos critérios. Indicará ser uma publicação de qualidade média, isto é, uma fonte útil de informação, mas terão algumas limitações, informações adicionais são necessárias.

- Qualidade ruim $<50 \geq 25 \%$ - a dimensão analisada (conteúdo, técnico e farmacológico) apresenta classificação (0) para a maioria dos critérios. Indicará que a publicação terá qualidade ruim, isto é, terão deficiências graves e não será uma fonte útil ou adequada de informações.

- Qualidade muito ruim $<25 \%$ - a dimensão analisada (conteúdo, técnico e farmacológico) apresenta classificação (0) para a maioria dos critérios. Indicará que a publicação terá qualidade muito ruim, isto é, terão deficiências muito graves e não será uma fonte confiável de informações.

Os sites foram classificados quanto à sua origem: Institucional, Leigos e Profissional de saúde.

Os resultados obtidos com a aplicação das ferramentas - qualidade das informações globais em sites para a saúde e avaliação da qualidade da informação sobre utilização de antidepressivos de cada site selecionado para o estudo foram tabulados no programa Microsoft Office Excel. 


\subsection{Análise estatística}

Para a estatística descritiva dos dados com distribuição paramétrica, foram calculados, média e desvio padrão. Para comparação da qualidade geral dos sites quanto à origem (especializado em saúde x não especializado em saúde; com contribuição de profissional de saúde x sem contribuição de profissional de saúde; institucional x leigo) foi utilizado o teste Kruskall-Wallis, considerando intervalo de confiança de $95 \%$. Os cálculos foram feitos na calculadora online disponível em https://www.socscistatistics.com/tests/kruskal/default.aspx.

Para classificação do conteúdo específico para depressão foi empregada a classificação própria da ferramenta, sendo considerados de boa qualidade os sites cuja pontuação se situou no quartil superior, de qualidade moderada os que tiveram pontuação situada na faixa do terceiro quartil, ruins os situados no segundo quartil e muito ruins os que tiveram pontuação situada no quartil inferior (primeiro quartil). Para a correlação entre os resultados dos dois avaliadores que realizaram as análises foi feito o teste de concordância kappa, sendo que valores abaixo de 0,40 representam baixa concordância, valores situados entre 0,41 e 0,60 representam concordância mediana aceitável, valores entre 0,61 e 0,80 representam forte concordância e valores acima de 0,81 representam concordância quase perfeita. Para o teste kappa foi utilizado o programa RealStatistics.

\section{Resultados}

\subsection{Sites selecionados}

Após exclusão dos sites de acordo com os critérios descritos, cinquenta sites foram avaliados respeitando a proporcionalidade de 34 sites para o buscador Google e 16 sites para o buscador Yahoo.

Tabela 1 - Quantidade e motivo da exclusão dos sites nas ferramentas de busca Google e Yahoo.

\begin{tabular}{c|c|c}
\hline $\begin{array}{c}\text { Número de sites } \\
\text { excluídos } \\
\text { (buscador Google) }\end{array}$ & $\begin{array}{c}\text { Número de sites excluídos } \\
\text { (buscador Yahoo) }\end{array}$ & $\begin{array}{c}\text { Motivo da } \\
\text { Exclusão }\end{array}$ \\
\hline 1 & 0 & Vídeo \\
\hline 2 & 43 & Duplicado \\
\hline 4 & 4 & Bulário \\
\hline 33 & 12 & Não específicos \\
\hline 6 & 2 & PDF/Artigo \\
\hline 10 & 3 & Anúncios \\
\hline 7 & 2 & Link inválido \\
\hline 1 & 3 & \\
\hline Total 64 & Total 69 & \\
\hline
\end{tabular}

Fonte: Autores.

\subsection{Classificação quanto à origem dos sites}

A maioria dos sites avaliados (84\%) pertencia a instituições, públicas ou privadas. Apenas $6 \%$ dos sites eram de leigos e $10 \%$ de profissionais de saúde. Foi observada a presença do selo HONCode em $6 \%$. Dos cinquenta sites, $70 \%$ se auto referiam como da área de saúde e 74 \% citavam a participação de profissional de saúde na produção da informação veiculada (Tabela 2). 
Research, Society and Development, v. 10, n. 10, e38101018509, 2021

(CC BY 4.0) | ISSN 2525-3409 | DOI: http://dx.doi.org/10.33448/rsd-v10i10.18509

Tabela 2 - Distribuição das características gerais dos sites, $\mathrm{N}=50,2020$.

\begin{tabular}{|c|l|c|}
\hline \multicolumn{1}{|c|}{ Aspecto analisado } & & N \\
\hline \multirow{2}{*}{ Origem } & Institucional & 42 \\
\cline { 2 - 3 } & Prof.de saúde & 3 \\
\cline { 2 - 3 } Site de saúde & Leigo & 35 \\
\hline \multirow{2}{*}{ Cita profissional de saúde } & Sim & 15 \\
\cline { 2 - 3 } & Não & 13 \\
\hline \multirow{2}{*}{ Selo HON CODE } & Sim & 37 \\
\cline { 2 - 3 } & Não & 3 \\
\hline \multirow{2}{*}{ Classificação Geral } & Sim & 47 \\
\cline { 2 - 3 } & Não & 0 \\
\hline \multirow{2}{*}{ Classificação Conteúdo Depressão } & Alta & 16 \\
\cline { 2 - 3 } & Moderada & 32 \\
\cline { 2 - 3 } & Ruim & 24 \\
\cline { 2 - 3 } & Muito ruim & 4 \\
\cline { 2 - 3 } & Muim & 4 \\
\cline { 2 - 3 } & Muito ruim & \\
\hline
\end{tabular}

Fonte: Autores.

\subsection{Avaliação da qualidade geral das informações}

Foram aplicados os questionários para avaliar as dimensões conteúdo, técnica e design.

Para a ferramenta de Mendonça et al. (2015), a concordância na classificação geral dos sites entre os avaliadores F e R foi quase perfeita $(\mathrm{k}=0,95)$.

Nenhum dos sites analisados foi classificado com qualidade alta, $32 \%$ foram classificados com qualidade moderada, $64 \%$ com qualidade ruim e $4 \%$ com qualidade muito ruim (Figura 1). 
Figura1 - Desempenho dos sites nas dimensões conteúdo, técnica e design.

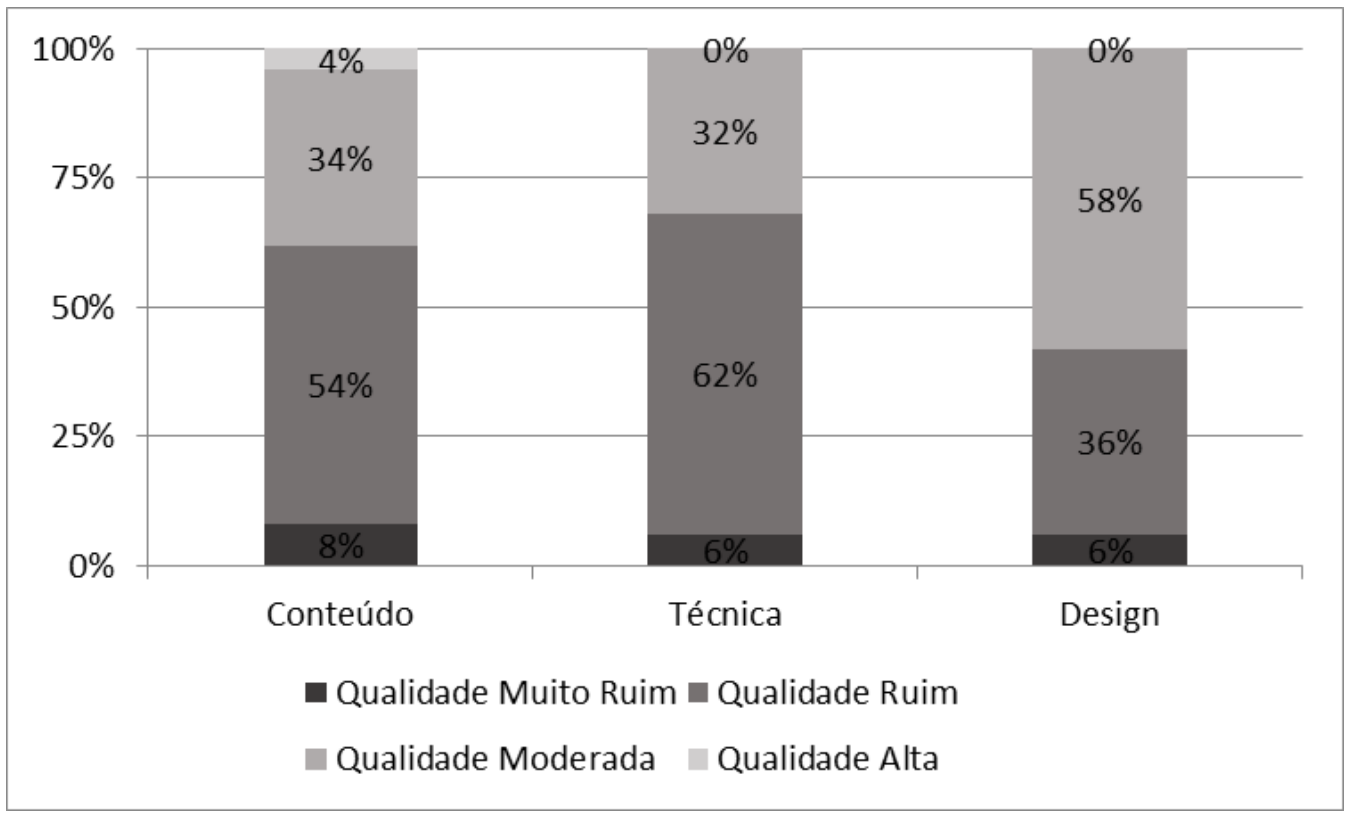

Fonte: Autores.

Considerando-se apenas a dimensão conteúdo, apenas $4 \%$ dos sites foram classificados com qualidade alta. Nesta dimensão, apresentaram problemas em relação à presença de informação de diagnóstico (16 \%), informação de prevenção (14 \%), contato para informação adicional $(10 \%)$ e fontes das informações disponíveis (26\%). Como ponto positivo, $92 \%$ apresentaram as fontes do texto em tamanho adequado (Figura 2).

Figura 2 - Desempenho de alguns indicadores em destaque na dimensão conteúdo.

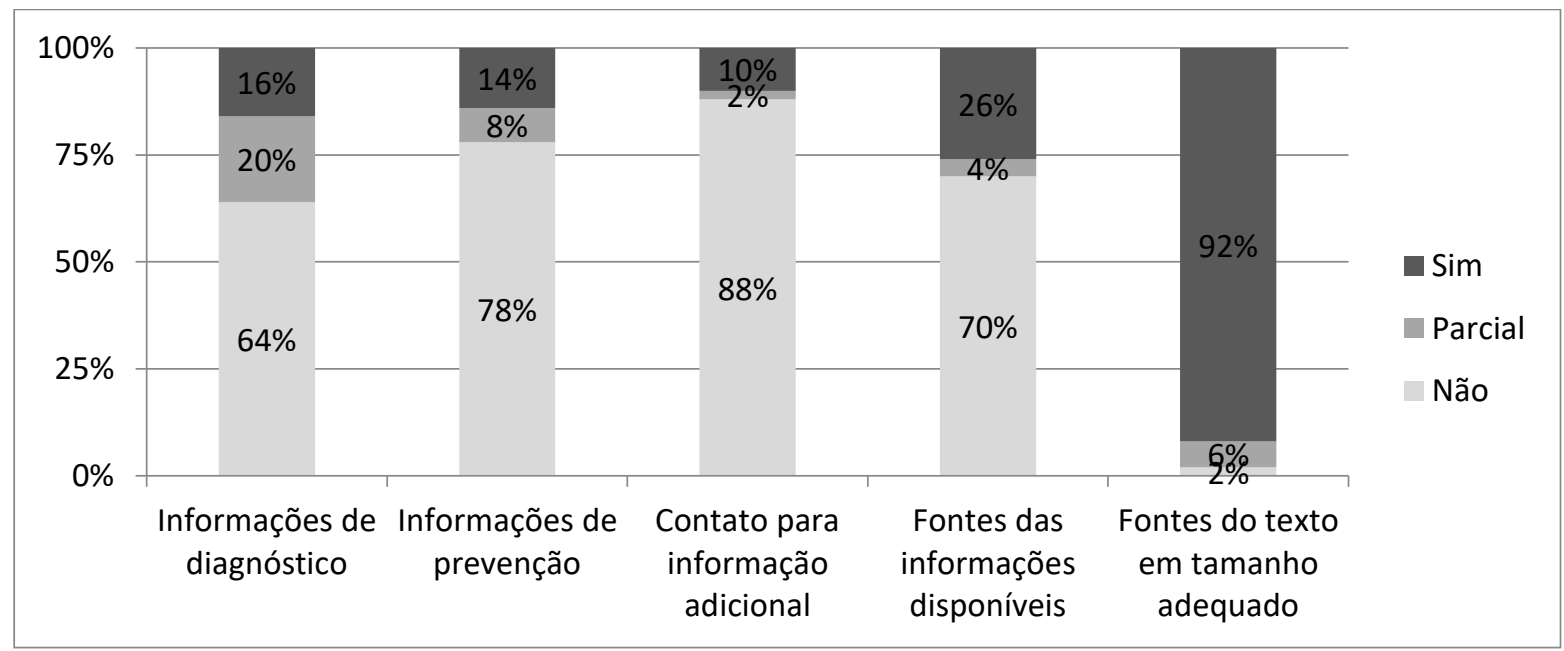

Fonte: Autores.

Na dimensão técnica, $62 \%$ dos sites foram considerados de qualidade ruim. Entre os critérios que se destacaram, encontram-se a ausência da data de criação do site (88 \%), e ausência da data de atualização do site (70 \%). Apesar de $74 \%$ apresentarem o autor da informação, $50 \%$ não apresentaram as credenciais desses autores. Em $88 \%$ dos sites foram observados anúncios, que refletem a presença de elementos de distração do usuário. Como ponto positivo desta dimensão, destaca-se a notificação da necessidade de consulta médica por $74 \%$ dos sites. Já a presença de ferramentas interativas como redes sociais e 
comentários $(88 \%)$ e fale conosco $(88 \%)$, se destacaram na dimensão técnica com suporte técnico adequado e boa interatividade com o usuário (Figura 3).

Figura 3 - Desempenho de alguns indicadores em destaque na dimensão técnica.

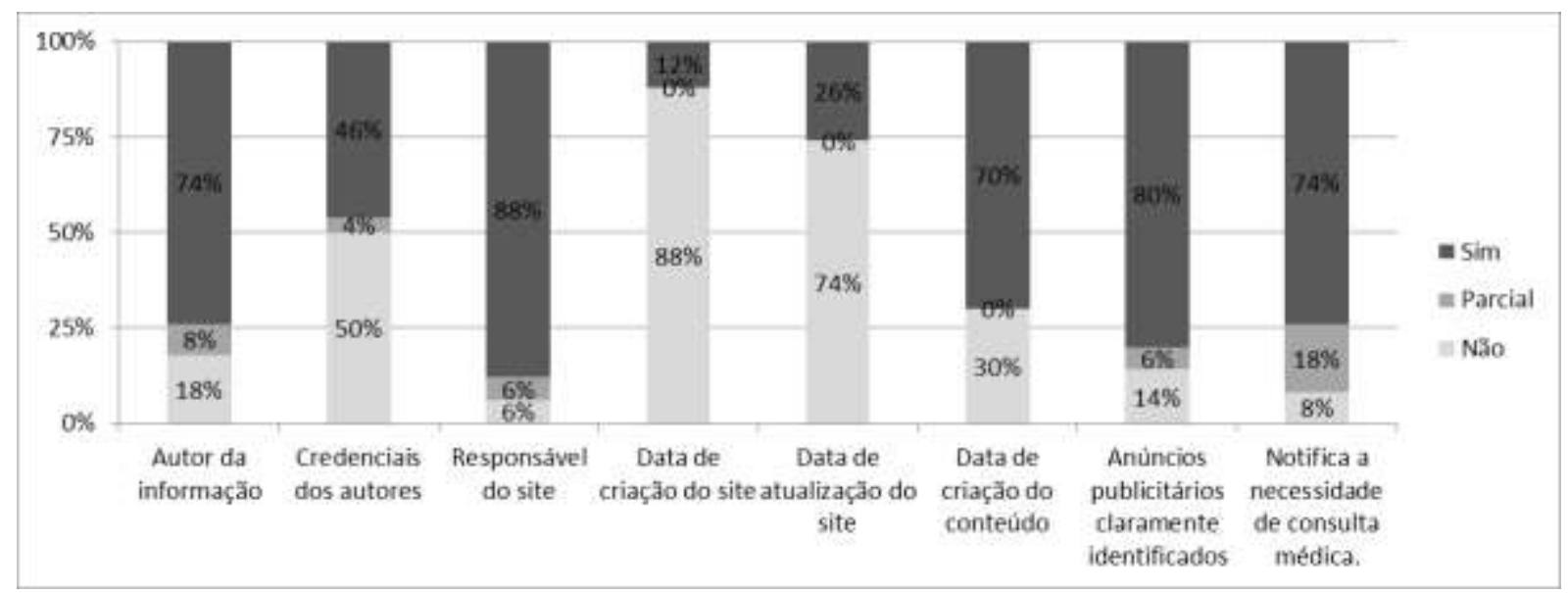

Fonte: Autores.

Na dimensão design $58 \%$ dos sites foram classificados com qualidade moderada. Critérios como fácil navegação (76\%), design agradável e limpo (80\%), ferramenta de busca (84\%) e acessível em mais outros dispositivos como celular $(98 \%)$, indicaram a facilidade de uso e navegação do site. Por outro lado, aspectos como ausência de mecanismos para aumentar a fonte $(94 \%)$ e ausência do recurso de barra de acessibilidade (94\%) estiveram presentes na quase totalidade dos sites (Figura 4).

Figura 4 - Desempenho de alguns indicadores na dimensão design.

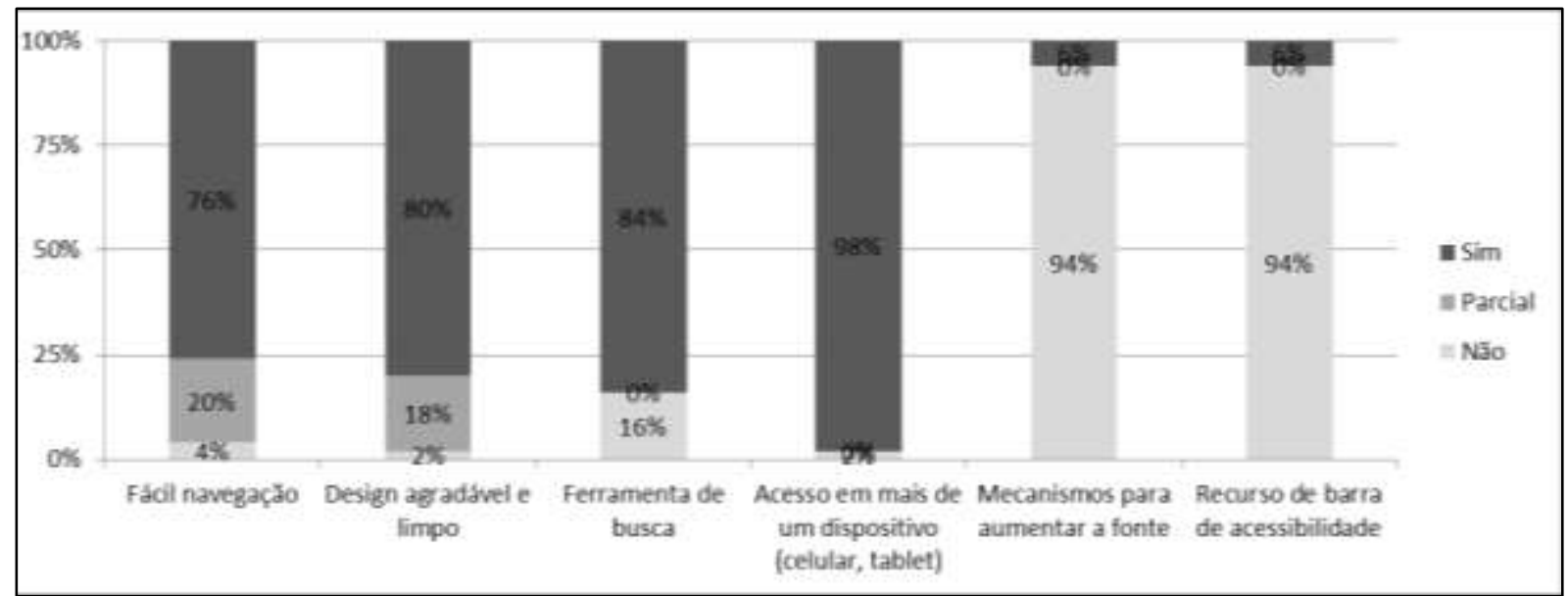

Fonte: Autores.

Ao se comparar a classificação geral dos sites especializados em saúde e aqueles que não são especializados, não se observou diferença estatisticamente significativa ( $\mathrm{p}=0,48479)$. A mesma situação foi observada ao se comparar os sites que citam a participação de um profissional de saúde na elaboração do conteúdo e os que não citam esta participação (p=0,1845) e ao comparar os sites institucionais e os demais $(\mathrm{p}=0,97889)$. 


\subsection{Avaliação da qualidade das informações sobre Depressão}

O nível de concordância para esta ferramenta proposta por Griffiths et al. (2005), entre os avaliadores F e T, se mostrou moderado $(\mathrm{k}=0,49)$.

Uma parcela importante ( $46 \%$ ) foi classificada com qualidade muito ruim, $40 \%$ com qualidade ruim, $6 \%$ com qualidade moderada. Apenas $8 \%$ dos sites foram considerados com qualidade alta.

Apenas o critério: "O perfil de efeitos colaterais varia para diferentes antidepressivos", esteve presente em mais da metade dos sites (76 \%). Alguns itens como (i): "Os antidepressivos são todos igualmente eficazes" e (ii): "a taxa de desistência é a mesma para diferentes antidepressivos" chegaram a estar ausentes em $98 \%$ e $94 \%$ dos sites, respectivamente (Tabela 3).

Tabela 3 - Avaliação dos sites sobre o conteúdo da depressão.

\begin{tabular}{|c|c|c|}
\hline CONTEÚDO Griffiths e colaboradores (2005) & Ausência & Presenç \\
\hline 1. A medicação antidepressiva é um tratamento eficaz para o transtorno depressivo maior. & $52 \%$ & $48 \%$ \\
\hline 2.Os antidepressivos são todos igualmente eficazes. & $98 \%$ & $2 \%$ \\
\hline 3.A eficácia dos antidepressivos é de cerca de 50 a $60 \%$. & $80 \%$ & $20 \%$ \\
\hline 4.A recuperação psicossocial total pode levar vários meses. & $72 \%$ & $28 \%$ \\
\hline 5.A taxa de desistência é a mesma para diferentes antidepressivos. & $94 \%$ & $6 \%$ \\
\hline 6.0 perfil de efeitos colaterais varia para diferentes antidepressivos. & $24 \%$ & $76 \%$ \\
\hline 7.A escolha do antidepressivo deve depender de fatores individuais do paciente (por exemplo, presença de com & $54 \%$ & $46 \%$ \\
\hline 8.Antidepressivos não são viciantes. & $78 \%$ & $22 \%$ \\
\hline 9.É necessário um estudo de 6 semanas com a dose total antes que um medicamento apresente falha e possa ter & $66 \%$ & $34 \%$ \\
\hline 10.Um medicamento de segunda linha provavelmente deve pertencer a uma classe diferente de antidepressivo. & $78 \%$ & $22 \%$ \\
\hline 11. Deve ser considerado melhor, o tratamento de continuação na mesma dose por pelo menos 4-6 meses. & $66 \%$ & $34 \%$ \\
\hline $\begin{array}{l}\text { 12. A síndrome de descontinuação pode ocorrer com a interrupção abrupta de qualquer antidepressivo, de } \\
\text { modo que os antidepressivos não devem ser parados de repente. Onde possível, antidepressivos devem ser } \\
\text { retirados ao longo de } 4 \text { semanas, a menos que haja razões médicas urgentes para interromper o medicamento } \\
\text { mais rapidamente. (Para marcar } 1 \text {, é necessário salientar que a cessação abrupta pode causar síndrome de } \\
\text { descontinuação e que os antidepressivos não devem ser parados de repente). }\end{array}$ & $52 \%$ & $48 \%$ \\
\hline $\begin{array}{l}\text { 13. A erva de São João parece ser tão eficaz quanto os antidepressivos tricíclicos e causa menos efeitos } \\
\text { colaterais, mas pouco se sabe sobre quaisquer efeitos adversos a longo prazo. }\end{array}$ & $82 \%$ & $18 \%$ \\
\hline 14. A terapia cognitiva pode ser um tratamento eficaz para a depressão. & $64 \%$ & $36 \%$ \\
\hline $\begin{array}{l}\text { 15. A terapia comportamental cognitiva é pelo menos tão eficaz quanto o tratamento medicamentoso na } \\
\text { depressão leve a moderada. }\end{array}$ & $68 \%$ & $32 \%$ \\
\hline $\begin{array}{l}\text { 16. A terapia comportamental cognitiva pode ser valiosa para pessoas que respondem ao conceito de cognição } \\
\text { terapia comportamental, preferem tratamento psicológico a antidepressivo, não responderam a terapia }\end{array}$ & $76 \%$ & $24 \%$ \\
\hline 17. A solução de problemas pode ser eficaz para a depressão. & $72 \%$ & $28 \%$ \\
\hline $\begin{array}{l}\text { 18. O aconselhamento [genérico] provavelmente não é mais eficaz que o tratamento usual do clínico para } \\
\text { depressão. }\end{array}$ & $88 \%$ & $12 \%$ \\
\hline $\begin{array}{l}\text { 19. Informações escritas (geralmente baseadas em um modelo cognitivo de depressão) podem melhorar de } \\
\text { leve a moderada depressão. [Pontuação } 1 \text { se modelo cognitivo] }\end{array}$ & $96 \%$ & $4 \%$ \\
\hline 20. O exercício pode ser eficaz - sozinho ou como complemento de outros tratamentos. & $70 \%$ & $30 \%$ \\
\hline
\end{tabular}

Fonte: Autores.

\section{Discussão}

Embora seja importante ressaltar que, com os mecanismos empregados pelos buscadores na internet, diferentes pessoas possam ter acesso a resultados diferentes usando os mesmos termos de busca, foi possível identificar problemas na qualidade da informação sobre depressão e antidepressivos em sites na língua portuguesa. Ainda que tenha sido observado certo grau de subjetividade na avaliação da qualidade do conteúdo específico para depressão com a utilização da ferramenta proposta por Griffiths et al. (2005), os resultados mostraram que nenhum dos cinquenta sites analisados, mostrou ter qualidade alta.

A baixa qualidade dos sites e das informações disponíveis na língua portuguesa na internet, tanto sobre a depressão quanto sobre os medicamentos utilizados em seu tratamento. Este resultado está de acordo com diversos estudos que apontam situação semelhante para diferentes temas em saúde (Buote et al., 2016; Pereira Neto et al., 2017; Paolucci et al., 2017), inclusive 
os relacionados à saúde mental e medicamentos a ela relacionados (Griffiths et al., 2017; Ma et al., 2017; Rathod et al., 2019; Ramos et al., 2020).

Pereira Neto et al. (2017) avaliando a qualidade da informação sobre dengue, observaram que nenhum dos 20 sites analisados alcançou boa classificação de acordo com os critérios utilizados. Ramos et al. (2020), avaliando a qualidade da informação sobre benzodiazepínicos, observaram que $56,3 \%$ dos 80 sites analisados foram classificados como deficientes ou muito deficientes.

No entanto, Garcia et al. (2018), estudando a qualidade da informação sobre vírus do papiloma humano, apontam boa qualidade da informação disponibilizada na internet, mostrando o potencial da internet para o empoderamento dos usuários desde que a qualidade da informação seja garantida.

Ainda segundo Mendonça et al. (2015), ao elaborar o conteúdo de um site, deve-se utilizar uma linguagem clara e acessível de forma que qualquer leitor possa compreender o que está sendo lido. Os resultados mostram que a linguagem se mostrou adequada ao público de destino em $64 \%$. No entanto, a qualidade da informação veiculada não se mostra a desejada.

Ao visitar um site, o usuário deve ser apresentado de forma clara ao seu objetivo devendo ser capaz de tirar suas dúvidas, fazer reclamações e dar sua opinião. Aspectos importantes como a segurança, credibilidade e a preocupação com a questão comercial devem se juntar ao aspecto ético do gestor da informação. A privacidade do usuário deve ser garantida e o site deve solicitar permissão para coletar qualquer informação pessoal. A interatividade, e o devido suporte ao usuário são, portanto, critérios de qualidade da dimensão técnica. Também nesta dimensão nenhum dos sites analisados apresentou a totalidade destes itens e mais da metade (62\%) foram classificados como de qualidade ruim.

A dimensão design confere critérios que indicam a facilidade de uso, de navegação e acessibilidade de acordo com as necessidades e expectativas dos usuários, reunindo critérios de usabilidade e acessibilidade para deficientes visuais, auditivos e motores. Os problemas encontrados nos critérios: ausência de mecanismos para aumentar a fonte (94 \%) e ausência do recurso de barra de acessibilidade (94\%), deixam clara a falta de acessibilidade à informação destes sites por pessoas com estas necessidades especiais. Situação semelhante foi apontada por Gibson et al. (2019). Um site mal projetado pode tornar as informações difíceis de ler e absorver, reduzindo a clareza. Nenhum dos sites foi classificado com qualidade alta, porém, $58 \%$ foram considerados de qualidade moderada nesta dimensão.

Os resultados deste estudo não mostraram diferença estatisticamente significativa entre a classificação geral e a origem dos sites. Situação semelhante foi apontada por Barbosa et al. (2007), que observaram que a qualidade não estava correlacionada o tipo de responsável pelo site.

Dos cinquenta sites analisados, $46 \%$ tiveram a qualidade do conteúdo sobre depressão, classificada como muito ruim. Este resultado é consistente com o observado por Griffiths et al. (2005) utilizando a mesma ferramenta de análise de conteúdo de depressão empregada neste trabalho. Os autores também consideraram a qualidade da informação baixa.

\section{Conclusão}

Os resultados confirmam a baixa qualidade da informação disponibilizada na Internet sobre depressão e antidepressivos. Alguns sites forneceram informações úteis a respeito de aspectos específicos da depressão, mas no geral a qualidade foi ruim. Há uma necessidade clara de melhorar a cobertura e a precisão do conteúdo, reforçando a discussão mundial sobre a necessidade de regulamentação dos conteúdos relacionados à saúde disponíveis na internet.

Desta forma, torna-se crucial a regulamentação da informação presente nos sites e a aplicação de selos de qualidade no Brasil, para que o usuário saiba em quais informações pode confiar, evitando o uso irracional de medicamentos e os graves problemas de saúde, que podem ocorrer como consequência da ausência ou baixa qualidade da informação disponibilizada. 
É interessante que estudo semelhante possa avaliar a informação veiculada em redes sociais como o facebook, youtube e instagram, por exemplo, sobre o mesmo tema. Da mesma forma, é interessante investigar a compreensão dos usuários sobre estas informações.

\section{Referências}

Barbosa, A. L., \& Martins, E. N. (2007). Evaluation of Internet websites about floaters and light flashes in patient education. Arquivos Brasileiros de Oftalmologia [online]. (70) 839-843.

Barros, M. B. de A.et al. (2020). Relato de tristeza/depressão, nervosismo/ansiedade e problemas de sono na população adulta brasileira durante a pandemia de COVID-19. Epidemiologia e Serviços de Saúde [online]. (29)e2020427.

Buote, R. D., Malone, S. D., Bélanger, L. J., \& McGowan, E. L. (2016). Quality and accuracy of publicly accessible cancer-related physical activity information on the Internet: a cross-sectional assessment. European journal of cancer care, 25(5), 795-805.

Caldeira, F. H. (2015). O mecanismo de busca do Google e a relevância na relação sistema-usuário. Letrônica, 8(1), 91-106.

Charnock, D., Shepperd, S., Needham, G., \& Gann, R. (1999). DISCERN: um instrumento para julgar a qualidade da informação escrita sobre a saúde do consumidor nas escolhas de tratamento. Journal of epidemiology and community health, 53 (2), 105-111.

Esteves, F. C, \& Galvan, A. L. (2006). Depressão numa contextualização contemporânea. Aletheia, (24), 127-135.

Garbin, H. B. R., Pereira Neto, A. F., \& Guilam, M. C. R. (2008). A internet, o paciente expert e a prática médica: uma análise bibliográfica. Interface Comunicação, Saúde, Educação [online]. (12), 579-588.

Garcia, R., Matias, M., Bastos, L., Bastos, R., \& Santos Koehler, F. (2018). Qualidade da informação em saúde: um estudo sobre o vírus do papiloma humano (HPV) em websites brasileiros. Revista Eletrônica de Comunicação, Informação e Inovação em Saúde, 12(1).

Gibson, J. M. E., Ellis, R. K., \& Jones, S. P. (2019) 'Dr Google' Will See You Now! Uma revisão de informações online ao consumidor sobre medicamentos anticoagulantes e antitrombóticos para prevenção de derrame recorrente. Journal of Consumer Health on the Internet, (23) 1-12.

Griffiths, K., Tang, T., Hawking, D., Christensen, H. (2005). Automated Assessment of the Quality of Depression Websites. J Med Internet Res, (7)e59.

Griffiths, K. M., \& Christensen, H. (2000). Quality of web based information on treatment of depression: cross sectional survey. BMJ (Clinical research ed.), 321(7275), 1511-1515.

Hirata, D. M., Queiroz, N. R., Souza, R. C., Oliveira, L. B., Martins, W. A. (2010). Qualidade da Informação na Internet sobre Coronariopatia. Rev Bras Cardiol, (23)39-46.

Lissman, T. L., \& Boehnlein, J. K. (2001). A critical review of internet information about depression. Psychiatric services (Washington, D.C.), 52(8), 10461050 .

Ma, Y., Yang, A. C., Duan, Y., Dong, M., \& Yeung, A. S. (2017). Quality and readability of online information resources on insomnia. Frontiers of medicine, 11(3), 423-431.

Mendonça, A., \& Neto, A. (2015). Critérios de avaliação da qualidade da informação em sites de saúde: uma proposta. Revista Eletrônica de Comunicação, Informação e Inovação em Saúde, (9).

Paolucci, R., Pereira Neto, A. F., Luzia, R. (2017). Avaliação da qualidade da informação em sites de tuberculose: análise de uma experiência participativa. Saúde debate (41)84-100.

Pereira, A. S. et al. (2018). Metodologia da pesquisa cientifica. UFSM. https://repositorio.ufsm.br/bitstream/handle/1/15824/Lic_Computacao_MetodologiaPesquisa-Cientifica.pdf?sequence.

Pereira Neto, A. F., Paolucci, R., Daumas, R. P., \& Souza, R. V. (2017). Avaliação participativa da qualidade da informação de saúde na internet: o caso de sites de dengue. Cien Saude Colet, (22)1955-1968.

Ramos, T. B., Bokehi, L. C., Oliveira, E. B., Gomes, M., Bokehi, J. R., \& Castilho, S. R. (2020). Informação sobre benzodiazepínicos: o que a internet nos oferece?. Ciencia \& saude coletiva, 25(11), 4351-4360.

Rathod, P., Ould Brahim, L., Belzile, E., \& Lambert, S. (2019). An evaluation of the suitability, readability, and quality of online resources for the selfmanagement of depression. Patient education and counseling, 102(5), 952-960.

Ribeiro, A. A. R., Melo, G. L. M. de, Costa, M. de S., Lima, M. F. de O., Torres, R. da S. G., \& Calil-Elias, S. (2021). Medicamentos analgésicos: Qual a qualidade da informação presente na Internet?. Research, Society and Development, (10)e25810817157.

Varady, N. H., Dee, E. C., \& Katz, J. N. (2018). International assessment on quality and content of internet information on osteoarthritis. Osteoarthritis and cartilage, 26(8), 1017-1026. 